\title{
1 Speed modulation of hippocampal theta frequency and power predicts water
}

2 maze learning

3

4 Calvin K. Young ${ }^{1}$, Ming Ruan ${ }^{1,2}$ and Neil McNaughton ${ }^{1}$

5

$6{ }^{1}$ Dept. Psychology and Brain Health Research Centre, University of Otago, Dunedin,

7 New Zealand

8 2Zhuhai Municipal Women's and Children's Hospital

10 Running Title: Movement speed and theta frequency

12 Corresponding Author:

13 Calvin K. Young

14 Department of Psychology,

15 University of Otago,

16 PO BOX 56, Dunedin, New Zealand

17 ORCID: Neil McNaughton ID = https://orcid.org/0000-0003-4348-8221

18 ORCID: Calvin K Young ID = https://orcid.org/0000-0002-6130-8370

19 Telephone: +64-3-479-7621

20 Fax: $\quad+64-3-479-8335$

21 Email: $\quad$ calvin.young@otago.ac.nz 


\section{Abstract}

24 Theta oscillations in the hippocampus have many behavioural correlates, with the 25 magnitude and vigour of ongoing movement being the most salient. Many consider 26 correlates of locomotion with hippocampal theta to be a confound in delineating theta 27 contributions to cognitive processes. But, theory and empirical experiments suggest 28 theta-movement relationships are important if spatial navigation is to support higher 29 cognitive processes. In the current study, we tested if variations in speed modulation 30 of hippocampal theta can predict spatial learning rates in the water maze. Using 31 multi-step regression, we find the magnitude and robustness of hippocampal theta frequency versus speed scaling can predict water maze learning rates. Using generalised linear models, we also demonstrate that speed and water maze learning are the best predictors of hippocampal theta frequency and power. Theta oscillations recorded from the supramammillary area showed much weaker, or non-existent, relationships, which supports the idea that hippocampal theta has specific roles in speed representation and spatial learning. Our findings suggest movement-speed correlations with hippocampal theta frequency may be actively used in spatial learning.

\section{Introduction}

41 The functional significance of hippocampal theta $(4-12 \mathrm{~Hz})$ oscillations has remained 42 an enigma since its initial characterisation (Green and Arduini, 1954). While the 43 mechanisms of its generation are still incompletely understood (Bland, 1986; 44 Buzsaki, 2002), the integrity of medial septal afferents (Brugge, 1965; Winson, 1978; 45 Rawlins et al., 1979) appears to be crucial (but see Goutagny et al., 2009). 46 Hippocampal theta has many behavioural correlates (Vanderwolf, 1969; Buzsaki, 2005; Young, 2011; Korotkova et al., 2018), the most salient among them is its linear scaling with the speed of ongoing locomotion, in both frequency (McFarland et al., 1975; Slawinska and Kasicki, 1998; Jeewajee et al., 2008; Wells et al., 2013; Aghajan et al., 2017) and power (Rivas et al., 1996; Maurer et al., 2005; Watrous et al., 2011; Ahmed and Mehta, 2012). Despite various replications in independent

52 laboratories, linear locomotion speed and hippocampal theta correlation can be 53 highly variable between animals and recording sessions (Richard et al., 2013; 54 Hernandez-Perez et al., 2016). It has been suggested that variability in the speed- 
55 theta correlations may be modulated by other functions that are dependent on theta 56 oscillations, such as learning and memory (Montgomery et al., 2009; Richard et al., 57 2013; Schmidt et al., 2013; Belchior et al., 2014; Hernandez-Perez et al., 2016).

58 Certainly, abolishing hippocampal theta oscillations do not result in the abolishment 59 of movement, but is correlated to spatial learning deficits (Winson, 1978; 60 McNaughton et al., 2006). Electrically driving theta-paced stimulation in the 61 hippocampus partially rescues hippocampal-dependent learning deficits 62 (McNaughton et al., 2006; Shirvalkar et al., 2010). Computational models integrating 63 spatial representations in the hippocampal-entorhinal circuit explicitly require speed 64 modulation of theta oscillations in their contribution to path integration and spatial 65 navigation (Burgess and O'Keefe, 2011), suggesting linear speed may be a partial correlate in its involvement in spatial navigation.

The linear theta-speed relationship can be degraded through uncoupling self-motion and actual translation through space, as indicated by studies using a running wheel (Czurko et al., 1999; Shin and Talnov, 2001), treadmills (Kuo et al., 2011), passive movement through carts (Terrazas et al., 2005) and virtual reality (Ravassard et al., 2013). Although swimming is natural and a normal mode of locomotion in rodents, past studies have indicated a lack of theta-speed relationship in swimming rats (Whishaw and Vanderwolf, 1973), presumably due to a disruption of proprioception associated with conventional ambulation on land. A more detailed study on hippocampal theta frequency and swimming speed have revealed an unreliable relationship at best (Hernandez-Perez et al., 2016) - although the use of conventional FFT-based spectral analysis in this study may have yielded less accurate estimates due to the non-stationary nature of brain field potentials and the time-frequency trade-off (Young and Eggermont, 2009).

Given the theoretical (Burgess and O'Keefe, 2011) and empirical (Brandon et al., 2011; Koenig et al., 2011; Bolding et al., 2019) support for hippocampal theta to contribute to spatial representation and spatial learning (McNaughton et al., 2006), we sought to carry out a more in-depth analysis on how hippocampal theta oscillations are related to locomotion/swimming parameters (i.e. speed and acceleration) in the water maze, and how the relationship may be linked to learning.

86 We show that hippocampal theta frequency and power, but not those recorded from

87 the supramammillary area, are differentially scaled with speed but less so with 
acceleration. The reliability and the magnitude of hippocampal theta versus swimming speed scaling can predict water maze learning rates. Together, our data support the notion that hippocampal theta-speed/acceleration relationships provide information relevant to path integration in water maze learning.

\section{Materials and methods}

\section{Subjects}

Eight male Sprague Dawley rats were acquired from the Department of Laboratory Animal Sciences at the University of Otago. These eight rats were taken from a larger dataset for a different experiment (McNaughton et al., 2006; Ruan et al., 2017), and were kept in a temperature and humidity controlled room with $12 \mathrm{hr}$ light/dark cycles (lights on at 6 a.m.). All rats had water and food access ad libitum throughout the experiment. The rats were given a ten-day acclimatisation period before surgical procedures. Relevant to the current study, stainless steel bipolar recording electrodes $(70 \mu \mathrm{m})$ were implanted in the hippocampus (AP: $-3.8 \mathrm{~mm}, \mathrm{ML}$ : $-2.5 \mathrm{~mm}$, DV: $-3.5 \mathrm{~mm}$ with tip separation of $1 \mathrm{~mm}$ ) and the supramammillary area (AP: $-4.8 \mathrm{~mm}$, ML: $-.9 \mathrm{~mm}$, DV: $-9.4 \mathrm{~mm}$ with tip separation of $.5 \mathrm{~mm}$ and implanted @ $6^{\circ}$ from vertical) under ketamine/ medetomidine $(75 \mathrm{mg} / \mathrm{kg}$ and $.5 \mathrm{mg} / \mathrm{kg}$, respectively) anaesthesia. Six anchor screws, one of which acted as the ground with uninsulated silver wire $(.25 \mathrm{~mm})$, held the implant with dental cement. A guide cannula and a stimulation electrode aimed at the medial septum and the fornix, respectively, were also implanted (McNaughton et al., 2006) but were not used in rats reported in the current study. Rats were given atipamezole $(2.5 \mathrm{mg} / \mathrm{kg})$ and postoperative analgesia at the end of the surgery and allowed to recover for at least ten days before any further manipulations. All procedures reported here were approved by the University of Otago Animal Ethics Committee (84/00 and 67/03).

\section{Water maze learning}

We implemented a one-day water maze training protocol (Pan and McNaughton, 1997). A circular black pool (diameter: $150 \mathrm{~cm}$; height: $35 \mathrm{~cm}$ ) was filled with $26^{\circ}$ ( \pm $2^{\circ} \mathrm{C}$ ) water to $25 \mathrm{~cm}$ deep. A black square $15 \mathrm{~cm}$ platform was placed $1.5 \mathrm{~cm}$ beneath the water and has a fixed position in the centre of the nominal "southeast" quadrant. The rats were connected to the recording system (see below) and allowed $40 \mathrm{~s}$ to find the platform. If successful, the rats remained on the platform for a further 
$12015 \mathrm{~s}$. If unsuccessful, the rats were guided to the platform. In either scenario, the

121 next trial started immediately after $15 \mathrm{~s}$ on the platform. A counterbalance sequence

122 of (NSWE ENSW WENS SWEN) 16 trials were administered. Position tracking and

123 water maze performance were carried out in HVS Image (HVS Image, UK).

124 Neurophysiological recordings, spectral analysis and statistics

125 Local field potentials (LFPs) were acquired through a custom-made unity gain

126 headstage and amplified (Grass P511K at 1-30 Hz band-pass). The data were

127 digitised $(100 \mathrm{~Hz})$ by a Micro1401 (CED, UK) with Spike2 software. Spectral analysis

128 was performed using the Chronux package (Bokil et al., 2010) with 5 tapers and a

129 numerical bandwidth of 3. All data analyses were carried out in Matlab (Mathworks,

130 Natick, MA). Statistics are reported as mean \pm standard error unless otherwise

131 stated. Raw $p$-values are reported for all statistical tests unless $p$-values are <.001,

132 where they are reported as $p<.001$. A false discovery rate was calculated at a

133 threshold of $p=.04$.

Instantaneous frequency and power

135 Raw LFP data were first z-scored prior to further processing. For frequency and 136 amplitude assessments, we opted to use analytic signal derivatives for 137 instantaneous frequency and power for near real-time estimation of these variables. 138 HPC and SuM LFPs were first band-passed (5-12 Hz, zero-phase) with an acausal 139 filter and then Hilbert transformed. The phase of the signals was smoothed by a $3^{\text {rd }}$ 140 order Savitzky-Golay filter and its derivative is taken as the frequency estimate. The 141 absolute value of the signal was taken as the envelope/power of the signal. Since 142 there was a ten-fold difference in acquisition rate between neurophysiological and 143 tracking data, both instantaneous frequency and envelope of the signals were binned 144 in 10 samples and the median value in the bins was taken as the final estimates 145 corresponding to a single tracking coordinate. The binned instantaneous frequency 146 and envelope are referred to as frequency and power, collectively as theta variables 147 throughout this report.

Position, speed, and acceleration

149 Position data were extracted from the text output from HVS Image (HVS Image, UK). 150 To minimise the misrepresentation of position, speed, and acceleration, we 
151 employed a 3-point median filter to correct minor tracking errors. However, we left

152 extended large jumps between points, no tracking data, and clear poor tracking data

153 as "no data" to avoid introducing bias to "instantaneous" position, speed and

154 acceleration estimates. Two trials from the dataset were missing too much data and

155 since they appeared to contain extensive mis-tracks by visual inspection were

156 discarded. Tracking data from one trial was missing and hence the trial was also

157 omitted. In total, 125 trials from eight rats were included in the final analyses.

158 Distance, speed, and acceleration were all calculated based on the known pixel-to-

159 centimetre relationship from the tracking coordinates.

160 In this study, we elected to use the conventional binned approach to examine the 161 relationship between theta frequency/power and speed/acceleration (Wells et al., 162 2013; Young and McNaughton, 2020). Although rats essentially swam continuously 163 inside the water maze, we decided to apply a low cut-off of $2.5 \mathrm{~cm} / \mathrm{s}$ as in previous 164 studies to: 1) facilitate direct comparisons to speed-theta relationships for land 165 locomotion and; 2) to exclude periods where rats vigorously attempt to climb up the 166 maze wall without spatial translation immediately after being placed into the pool 167 during early stages of learning. In addition, we excluded the first $2 \mathrm{~s}$ of data from 168 each trial to eliminate tracking errors relating to the experimenter placing rats into the 169 pool, as the tracking was triggered by any high-contrast entity entering the camera's 170 field of view. In sum, we employed $2.5 \mathrm{~cm} / \mathrm{s}$ speed bins across 5 to $45 \mathrm{~cm} / \mathrm{s}$ and 0.5 $171 \mathrm{~cm} / \mathrm{s} / \mathrm{s}$ acceleration bins across -3 to $3 \mathrm{~cm} / \mathrm{s} / \mathrm{s}$ for speed and acceleration estimates. 172 These are collectively termed kinematic variables throughout the current study.

\section{Spatial distribution and cross-correlations}

174 Spatial distributions of kinematic and theta variables were constructed by first 175 converting the value found at each coordinate into a "map" for each trial, where 176 repeated samples of the same variable at the same coordinates represented by the 177 average found in the spatial bin. Each raw spatial bin approximated a $20 \times 20 \mathrm{~cm}$ 178 area in the pool. All matrices from all trials were averaged to form a single map for 179 each variable, convolved with a $3 \times 3$ gaussian filter and up-sampled 3 fold to yield a 180 final map for 2D cross-correlation analysis. 
182 For linking theta variables to kinematic variables, we took the averaged theta 183 variables across all available trials from each rat as primary data. Linear regression was applied to each individual rat's data, as well as at the population level across rats. Slope and correlation coefficients were extracted from each fit.

Learning was quantified by extracting the exponential fit coefficient over the path length for each rat over the 16 trials. The slope and correlation coefficients of the theta frequency versus speed regressions from each individual rat were then regressed against their respective exponential fit coefficient of water maze performance over time. We used these series of regressions to demonstrate the robustness and reliability of theta frequency versus speed relationship with water maze learning.

\section{Instantaneous estimate of water maze performance}

194 To provide an "instantaneous" estimate of water maze performance, we introduced a performance metric taking into account the direction of the rats' trajectory, distance to the platform and normalised elapsed time in the trial. Particularly, we took the angle differences between each pair of tracking points to estimate the change in trajectory angle $\left(\theta_{\text {point }}\right)$, and the angle differences between each tracking point to the point representing the centre of the platform $\left(\theta_{\text {platform}}\right)$. The absolute difference between these two angles (i.e. point-to-point and point-to-platform) yielded a value between 0 and $\pi$ for headings towards the platform and $180^{\circ}$ away, respectively. This angle variable is then multiplied by the distance from the point to the platform

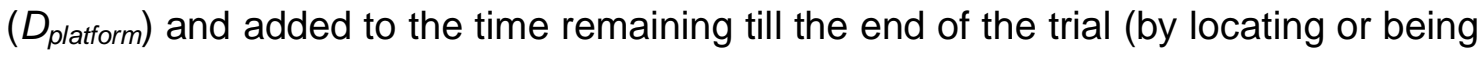
guided to the platform; $\left.T_{\text {end }}\right)$ :

$$
\text { Performance }=\left|\left(\theta_{\text {point }}-\theta_{\text {platform }}\right)\right| * D_{\text {platform }}+T_{\text {end }}
$$

This metric favours correct head trajectories towards the platform over the absolute distance from the platform to account for varied starting points (i.e. distances from 208 the hidden platform) for each trial. The addition of time remaining in the trial offsets spurious trajectory angles toward the hidden platform during the search.

\section{Generalised linear model}

211 To gauge the individual and combined contribution of swimming kinematics and 212 trajectory (i.e. performance) to theta measures, we entered z-scored binned 
213

swimming speed, binned acceleration and the performance metric as predictor variables so such:

$$
\theta_{\text {frequency } / \text { power }}=\beta_{\text {constant }}+\beta_{\text {speed }}+\beta_{\text {acceleration }}+\beta_{\text {performance }}+\mathcal{E}
$$

We extracted the fit parameters (i.e. slopes; standardised $\beta$, or $z \beta$ from hereon) for each rat and tested if the population averages are significantly different from zero with Student's t-tests.

\section{Histology}

At the end of the experiments, rats were euthanised with a lethal dose of sodium pentobarbital and transcardially perfused with physiological saline followed by $10 \%$ formalin in saline solution. After a minimum of $24 \mathrm{hr} 10 \%$ formalin fixation, the brains were dehydrated with $30 \%$ sucrose in $10 \%$ formalin solution until saturation and sectioned at $90 \mu \mathrm{m}$ on a freezing microtome. Electrode tracks were reconstructed from digitised Nissl stained sections.

\section{Results}

Continuous hippocampal and supramammillary theta in the water maze

We were able to confirm the placement of bipolar electrodes to have predominantly targeted the CA1 region of the hippocampus (Figure 1A, left panel) and the supramammillary area (Figure $1 \mathrm{~A}$, right). Both areas showed strong, continuous theta rhythmicity during swimming in the water maze by visual inspection (Figure 1B, left panels) and spectral analyses (Figure 1B, right panels). The presence of high amplitude, continuous theta oscillations throughout swimming circumvents the possibility our analytic signal-derived theta variables contain erroneous estimates of non-theta states.

\section{Spatial distribution of locomotion and theta parameters}

To examine how theta oscillations relate to behaviour in the water maze, we first mapped theta variables (i.e. frequency and power) and kinematic measures (i.e. speed and acceleration) in the pool. In general, there do not appear to be any differences between HPC and SuM theta frequency distributions at the periphery but 
242 HPC theta frequency may be lower (Figure 2A, left) in the inner annulus while SuM 243 theta frequency appears to be higher (Figure 2A, right). For theta power, the HPC 244 seems to have higher power in general (Figure 2B, left) compared to SuM (Figure $2452 \mathrm{~B}$, right). Speed (Figure 2C, left) distribution indicates low speeds in the target 246 quadrant, particularly around the platform itself, which is consistent with qualitative 247 observations that rats generally slow down near the hidden platform as part of the 248 searching strategy. The speed distribution also suggests that the fastest speeds are 249 observed in the "western" half of the pool. Acceleration (Figure 2C, right) shows a 250 similar distribution to speed in that the highest changes are away from the periphery 251 and on the "western" side of the pool. The platform is surrounded by a ring of 252 deceleration, which is surrounded by a larger ring of acceleration, again corroborating qualitative observations of rats swimming quickly towards the platform and slow down as they approach the platform as a search strategy. As expected, the periphery of the pool had the highest occupancy (Figure 2D) as rats spent most of their actual time in the water initially in thigmotaxis. There is also a clear increase in occupancy around the hidden platform (but not "south-east" from it), again consistent with observed finer searching behaviour in the vicinity of the hidden platform once rats acquire its location.

While some general patterns of theta and swimming kinematic parameters can be qualitatively described, we performed spatial autocorrelation to see if additional features exist. Apart from the already described faster speeds from the "western" side of the pool towards the middle and high occupancy around the periphery, no other spatial autocorrelation features emerged (data not shown). We further explored potential similarities in spatial distribution across all theta and kinematic parameters in the pool using spatial cross-correlation (Figure $2 \mathrm{E}$ ). There is a pattern of negative spatial cross-correlation between HPC theta frequency with swimming speed $(r=$ .21) and acceleration $(r=.22)$. There is weak positive spatial correspondence between HPC and SuM theta measures ( $r=.21$ for frequency; $r=.38$ for power), as well between speed and acceleration $(r=.28)$. There is minimal correlation between SuM theta power with speed $(r=.1)$ or acceleration $(r=.02)$, while SuM theta 272 frequency showed no spatial correspondence with speed $(r=.09)$ but a negative 273 correlation with acceleration $(r=-.26)$ and HPC theta power distribution showed 
274 positive spatial correspondence with speed $(r=.24)$ and weak negative correlation

275 with acceleration $(r=-.13)$.

276

277

278

279

280

281

\section{Water maze learning}

Before examining the relationship of theta variables with swimming kinematics and water maze learning, we first characterise metrics for learning. The decrease of path length as training progressed was evident for all but one rat (Figure 3A). An exponential decay function was fit for all "learning curves" (Figure 3B) and the slope parameter was extracted as a measure for water maze learning rate in analyses described in the next sections. We also introduced a moment-to-moment index of water maze performance as a metric for learning (see Methods). In Figure 3C, an example metric for the whole $40 \mathrm{~s}$ at the beginning of the training session (i.e. trial 1) is depicted, with its corresponding trajectory mapped in Figure 3E. In the last trial, where rats exhibited much shorter path length to reach the hidden platform, the metric accounts for erroneous trajectories away from the hidden platform and regress towards zero (Figure 3D) as rats successfully locate the platform (Figure $3 F)$.

\section{Frequency and power correlates to speed}

In the water maze, all rats except for one showed a nominal positive correlation between swimming speed and HPC theta frequency (Figure 4A, left). More variable regression lines for SuM theta frequency versus swimming speed (Figure 4A, middle) suggest a lack of relationship between the two variables. At the population level, HPC theta frequency and swimming speed do share positive linear correlation $\left(r^{2}=.42, F(15)=10.98, p=.005\right)$ while a more modest negative correlation was observed for SuM theta frequency versus swimming speed $\left(r^{2}=.30, \mathrm{~F}(15)=6.57, p\right.$ $=.022)$. To explore if better linear scaling between theta frequency and swimming speed correlates to learning, we regressed the slope (Figure 4B, top panels) and $r^{2}$ (Figure 4B, bottom panels) of individual theta frequency versus swimming speed linear fits against learning (i.e. slopes of exponential fits from Figure 3B). Both the slope (Figure 4B, top left; $r^{2}=.54, \mathrm{~F}(6)=7.11, p=.037$ ) and $r^{2}$ (Figure 4B, bottom left; $\left.r^{2}=.71, F(6)=15.02, p=.008\right)$ showed better learning associated with stronger/more reliable theta frequency versus swimming speed scaling. A similar but much weaker trend can be seen for the SuM equivalents for both slope (Figure 4B, 
306 top right; $r^{2}=.22, \mathrm{~F}(6)=1.67, p=.236$ ) and $r^{2}$ (Figure $4 \mathrm{~B}$, top left; $r^{2}=.43, \mathrm{~F}(6)=$

$307 \quad 4.48, p=.079)$

308

309

310

The theta power versus swimming speed relationships in the HPC across all rats were consistently positive, except for one rat (Figure 4C, left). As with frequency, theta power versus swimming speed also yielded high variability in individual fits but mostly positive (Figure 4C, middle). At the population level, both $\operatorname{HPC}\left(r^{2}=.74, \mathrm{~F}(15)\right.$ $=43.44, p<.001)$ and SuM $\left(r^{2}=.47, \mathrm{~F}(15)=13.23, p=.002\right)$ theta power showed a positive correlation with swimming speed (Figure $4 \mathrm{C}$, right). Unlike with theta frequency, neither slope $\left(r^{2}=.007, \mathrm{~F}(6)=.04, p=.85\right)$ nor $r^{2}\left(r^{2}=.004, \mathrm{~F}(6)=.02, p\right.$ $=.88$ ) of theta power versus swimming speed linear regressions from the HPC correlated with water maze learning (Figure 4D, left panels). Slope $\left(r^{2}=.23, F(6)=\right.$ 1.83, $p=.22)$ and $r^{2}\left(r^{2}=.26, F(6)=2.06, p=.20\right)$ for SuM theta power versus swimming speed had better linear fits than their HPC counterparts, but were not statistically significant (Figure 4D, right panels). A summary between linear scaling of theta variables and their relationship with water maze learning are presented in Table 1.

\section{Frequency and power correlates to acceleration}

Individual linear fits for HPC theta frequency versus acceleration yielded mostly negative slopes with a single exception (Figure 5A, left). The slopes of linear fits in the SuM is also predominantly negative with more variability (Figure 5B, middle). Previous studies have shown both HPC theta frequency (Shin and Talnov, 2001) and power (Long et al., 2014) may show better positive correlation with deceleration than acceleration. Given the shallow slopes at the individual level linear regression, we opted to perform population level theta measure versus acceleration linear regressions separately for positive acceleration and negative acceleration, in addition to a single fit encompassing acceleration and deceleration. A summary of linear scaling of theta variables by negative, positive and overall acceleration is presented in Table 2. Overall linear regression of HPC theta frequency versus acceleration (Figure 5A, left) reveal a negative correlation $\left(r^{2}=.68, \mathrm{~F}(10)=23.09, p\right.$ $<.001)$, with negative acceleration $\left(r^{2}=.79, \mathrm{~F}(10)=19.04, p=.007\right)$ presumably contributing more to the negative slope than positive acceleration $\left(r^{2}=.02, F(10)=\right.$ $.09, p=.78)$. In the SuM, the overall fit has a marginal negative slope $\left(r^{2}=.24, F(10)\right.$ 
$338=3.55, p=.09)$ with theta frequency weakly correlating to the magnitude of negative $339\left(r^{2}=.19, \mathrm{~F}(10)=1.19, p=.33\right)$ and positive $\left(r^{2}=.47, \mathrm{~F}(10)=3.56, p=.13\right)$

340 acceleration. Since the overall fit provided the best fit compared to negative or 341 positive acceleration fits in both the HPC and the SuM, we regressed the individual 342 slope (Figure 5B, top left; $r^{2}=.01, \mathrm{~F}(10)=.08, p=.79$ ) and $r^{2}$ (Figure 5B, bottom left; $343 r^{2}=.37, F(10)=3.51, p=.11$ ) from the overall fits to water maze learning (i.e. slope 344 of the exponential fit over path length over time) and found no relationship. SuM 345 theta frequency versus acceleration fit slopes (Figure $5 \mathrm{~B}$, top right; $r^{2}=.65, F(10)=$ 346 11.36, $p=.02$ ) and $r^{2}$ (Figure 5B, bottom right; $r^{2}=.56, F(10)=7.66, p=.04$ ) correlated with water maze learning.

Both the HPC (Figure 5C, right) and SuM (Figure 5C, middle) show weak and variable associations between theta power and acceleration. At the population level, the data suggest a clear quadratic relationship between acceleration and theta power (Figure 5C, right). This observation is supported by much stronger association between negative $\left(r^{2}=62, \mathrm{~F}(10)=8.09, p=.04\right)$ or positive acceleration $\left(r^{2}=98\right.$, $F(10)=165.93, p<.001$ ) with HPC theta power alone, rather than a linear fit using the overall acceleration $\left(r^{2}<.001, F(10)=.09, p=.76\right)$. Although a similar relationship can be observed for SuM theta power and acceleration, the negative acceleration $\left(r^{2}=.85, F(10)=29.33, p=.003\right)$ yielded a better fit than the positive $\left(r^{2}\right.$ $=40, \mathrm{~F}(10)=2.62, p=.18)$, as well as the overall acceleration $\left(r^{2}=.06, F(10)=.67\right.$, $p=.43$ ). Given the relationships between acceleration and theta power are clearly not linear and appear to be quadratic, we performed linear fits on the absolute values of acceleration and extracted the slope and $r^{2}$ to regress against water maze learning estimate. Similar to HPC theta power relationships with speed, neither slope $\left(r^{2}<.05, \mathrm{~F}(10)=.30, p=.60\right)$ nor $r^{2}\left(r^{2}<.001, \mathrm{~F}(10)<.001, p=.99\right)$ of absolute acceleration fits correlated well with the rate of spatial learning (Figure 5D, right panels). The same is for the SuM, showing no correspondence between the slopes $\left(r^{2}=.09, \mathrm{~F}(10)=.60, p=.47\right)$ and $r^{2}\left(r^{2}=.02, \mathrm{~F}(10)=.13, p=.24\right)$ extracted from absolute acceleration and water maze learning (Figure $5 \mathrm{D}$, right panels). A summary between linear scaling of theta variables and their relationship with water maze learning are presented in Table 1. 
370 From our regression analyses, it appears both theta variables have better correlates

371 with speed rather than acceleration, and that how HPC theta frequency scaling with 372 swimming speed serve as a superior predictor for water maze performance 373 compared to other possible combinations. As an alternative way of characterising a 374 potential link between speed modulation on HPC theta frequency relating to learning, 375 as well as circumventing the need to perform multi-step regressions with 376 dimensionally reduced data (i.e. regressing fit parameters against another), we 377 performed GLM with binned speed, binned acceleration and our novel performance 378 metric as potential predictors for theta measures for each rat. For theta frequency versus swimming speed (Figure 6A), $z \beta$ were all positive for HPC except for one rat. As a group, the $z \beta$ were significantly different to zero $(\mathrm{t}(7)=3.05, p=.02)$. This was not the case for SuM theta frequency $(\mathrm{t}(7)=-.04, p=.68)$. Acceleration was a poor predictor for both HPC $(\mathrm{t}(7)=96, p=.38)$ and SuM $(\mathrm{t}(7)=-.53, p=.61)$ theta frequency. Interestingly, the water maze performance metric appears to be the best predictor for theta frequency in both the HPC $(\mathrm{t}(7)=5.85, p<.001)$ and the SuM $(\mathrm{t}(7)=2.59, p=.04)$.

For the same analyses relating to theta power, speed appeared to be a good predictor for both HPC $(\mathrm{t}(7)=2.99, p=.02)$ and SuM $(\mathrm{t}(7)=2.20, p=.06)$ theta power (Figure 6B). Acceleration is the best predictor for HPC theta power $(\mathrm{t}(7)=$ 3.59, $p=.009)$ but not for the SuM $(\mathrm{t}(7)=1.01, p=.35)$. Water maze performance also served as a good predictor for HPC theta power $(\mathrm{t}(7)=3.08, p=.02)$, but less so for the SuM $(\mathrm{t}(7)=1.96, p=.09)$.

\section{Discussion}

Much theory and empirical data suggest HPC theta oscillations, particularly concerning locomotion speed, have a role in spatial navigation (Montgomery et al., 2009; Burgess and O'Keefe, 2011; Richard et al., 2013; Schmidt et al., 2013;

396 Belchior et al., 2014; Hernandez-Perez et al., 2016; Korotkova et al., 2018). We used the rate of water maze learning as a functional test for spatial navigation. We were able to show: 1) HPC theta frequency and power scale linearly with swimming speed; 2) HPC theta frequency and power have opposite spatial distributions in the water maze; 3) more robust HPC theta frequency scaling with swimming speed is 
spatial navigation are the best predictors for HPC theta frequency; and, 5) swimming speed, acceleration and goal-directed spatial navigation all contribute to HPC theta power and frequency predictions. Our findings are consistent with the notion that HPC theta reflects inherent path integration abilities in individuals, which predict the rate of water maze learning.

We were able to show that swimming speed correlates to, and is a useful predictor of, HPC theta frequency. Previous studies have reported a lack of robust linear correlation between HPC theta frequency and swimming speed (Whishaw and Vanderwolf, 1971; Hernandez-Perez et al., 2016). However, the conclusions were either made qualitatively (Whishaw and Vanderwolf, 1971) or based on a longer $2 \mathrm{~s}$ window spectral estimate of theta frequency that was combined with an unknown undescribed treatment of tracking data at a higher rate (Hernandez-Perez et al., 2016). Since we employed "instantaneous" theta frequency and power derived from the analytic signal in our study, the increase in precision in the temporal domain may have unmasked inherent speed modulation of HPC theta frequency that exists in swimming animals. However, it is clear from converging studies that uncoupling movement speed from motor output results in the loss/flattening of the HPC theta frequency versus speed relationship (Czurko et al., 1999; Shin and Talnov, 2001; Terrazas et al., 2005; Kuo et al., 2011; Ravassard et al., 2013); it remains to be seen if movements at the same speeds between swimming and running animals would differ in the strength (i.e. slope) of the scaling function.

Our data indicate movement speed does partially account for variance in HPC theta frequency, but estimates of water maze learning appear to provide more predictive value. This is in line with previous studies, where GLM identified movement speed and location on the maze, but not acceleration, as robust predictors of HPC theta frequency and to a lesser extent, power, in spatial tasks (Montgomery et al., 2009; Schmidt et al., 2013). A different study examining HPC theta frequency versus movement speed relationships also revealed that the slope and $r^{2}$ were both correlated with spatial alternation performance (Richard et al., 2013). More indirectly, when movement speeds are matched, there is an apparent increase in HPC theta power and frequency correlating to correct spatial choices in a foraging task (Belchior et al., 2014). Our findings reported here are consistent with the idea that HPC-dependent task performance metrics are highly correlated with HPC theta 
435 frequency but less so with power. HPC theta frequency is known to be less variable

436 than power across the whole hippocampus (Maurer et al., 2005; Montgomery et al.,

437 2009; Hinman et al., 2011), hence it is not surprising measures of kinetics and

438 spatial learning correlated better with HPC theta frequency than power.

439 In our study, the behavioural context is one of spatial learning. It has been 440 documented previously that exposure to novel spatial environments reduces the 441 slope of the HPC theta frequency versus movement speed relationship (Jeewajee et 442 al., 2007; Wells et al., 2013). Unfortunately, limitations imposed by our study design 443 prevent a conclusive demonstration of increases in HPC theta frequency versus 444 speed slopes as a function of learning (i.e. progression from "novel" to "familiar" 445 environments). Due to the nature of the water maze learning task, less data become 446 available as animals learn to successfully locate the hidden platform. We were able 447 to detect a correlation between the slope of HPC theta frequency versus speed and 448 path length (data not shown). However, high variances in the direction (i.e. 449 positive/negative slopes) and value of fitted slope as a function of path length, 450 presumably driven by the paucity of data in shorter trials, prevented any useful 451 conclusions to be drawn. Future studies utilising probe trials at the end of training 452 would be useful in providing adequate data to investigate if increases in slope of the 453 HPC theta frequency versus swimming speed relationship emerges as animals 454 acquire the position of the hidden platform (i.e. as the novel spatial environment 455 becomes familiar). Relocation of the hidden platform would also be useful in 456 disambiguating effects of spatial novelty on HPC theta frequency versus speed 457 scaling and learning a new goal location.

458 We have demonstrated that the HPC theta frequency versus swimming speed 459 relationship can predict learning rates in the water maze. It is currently unclear if the 460 predictive value is restricted to swimming speeds, or whether it can be generalised to 461 the strength of HPC theta frequency versus movement speed on dry land. A crucial 462 experiment validating our proposed importance of HPC theta frequency versus 463 speed scaling in spatial cognition would be to examine if the strength of frequency 464 versus speed coupling on land is predictive of water maze learning rate in individual 465 animals. If so, it would suggest the slope and to a certain extent, measures of 466 goodness-of-fit (e.g. $\left.r^{2}\right)$ of HPC theta frequency versus speed relationship may serve 467 as a potential biomarker for spatial cognition and its deficits. In addition, the use of a 
multi-day instead of a single-day paradigm used in the current study would also provide further insight on if the relationships described here is generalisable in water maze learning and goal-directed spatial navigation and not dependent on unknown features of the single-day implementation.

A potential confound in our study is the effect of body temperature reduction in our single-day, 16 consecutive trials version of the water maze. Previous studies have shown a reduction in body temperature reduces HPC theta frequency (Whishaw and Vanderwolf, 1971; Wells et al., 2013). We have previously demonstrated that the effect of cooling on HPC theta frequency follows a strong exponential decay function (Pan and McNaughton, 1997), which is also the type of curve fitting we performed to assess learning rates in the current study. Given we did not monitor the rats' body temperature, how changes in body temperature throughout our water maze training impact on our conclusions cannot be accounted for. However, we note that our GLM analysis is not directly impacted by the exponential decrease in path length and body temperature as a function of time. With our novel real-time water maze performance metric, the GLM analysis suggested that our instantaneous performance metric can still predict changes in HPC theta frequency, independent of shared similarities between path length and body temperature time-courses.

Although the HPC theta frequency versus movement speed relationship is welldescribed, it is not the only speed representation available to the canonical spatial circuits. Speed information is also represented in firing rates (Kropff et al., 2015; Gois and Tort, 2018) and burst frequency (King et al., 1998) of single cells along the spatial circuit the HPC is a part of, and appear to coexist as a competing source of speed information in the medial entorhinal cortex (Hinman et al., 2016). Some experimental evidence suggests self-motion- and sensory-derived (mostly visual) signals may underlie the existence of multiple speed information in the spatial circuit (Campbell et al., 2018; Jayakumar et al., 2019). The nature of the speed information that the CA1 theta LFP carries is unclear; however, this theta appears to be essential for spatial representation in the HPC and medial entorhinal cortex (Brandon et al., 2011; Koenig et al., 2011; Bolding et al., 2019), and uncoupling of self-motion and actual movement appear to suppress its relationship with speed (Czurko et al., 1999; Shin and Talnov, 2001; Terrazas et al., 2005; Kuo et al., 2011; Ravassard et al., 2013). Of interest, there is no reported change in place cell firing rates in the water 
501 maze in relation to speed (Hollup et al., 2001a, b). Given local field potentials are

502 epiphenomena and a composite surrogate measure for local excitability, it is clear 503 from the current and other studies that HPC theta, particularly frequency, carries 504 speed-related information among other types. Additional experiments are needed to 505 tell if and how multiple speed codes in the HPC represent different speed 506 representations such as self-motion, perceived movement through space, and 507 physical effort related to movements.

508 Human HPC theta oscillations are also correlated to movement through space 509 (Ekstrom et al., 2005; Bush et al., 2017), mnemonic processes (Rutishauser et al., 510 2010) and spatial navigation (Bush et al., 2017). Importantly, the removal of salient 511 sensory cues in virtual reality environments lowers the frequency of human 512 hippocampal theta (Bohbot et al., 2017), which may reflect a decrease of the theta513 speed relationship as seen in rodents. Overall, there is a good correspondence 514 between human and rodent data on how HPC theta relates to movement kinematics 515 (Kunz et al., 2019). There is also evidence that such relationships can be exploited in 516 clinical settings to assess the effectiveness of anxiolytics in the HPC (Wells et al., 517 2013; Young and McNaughton, 2020) and beyond. An improved understanding of 518 how the HPC theta frequency versus speed relationship is generated, modulated 519 and utilised in the brain can provide fundamental insights to sensorimotor integration 520 (Bland and Oddie, 2001) in health and disease.

\section{Conclusions}

522 Previous studies have shown behavioural tasks can significantly modulate HPC 523 theta oscillations, beyond its correlations with movement speed. Our findings take a 524 step further in providing evidence to support the idea that HPC theta frequency 525 correlations with speed may be actively utilised to support spatial cognition. This 526 appears to be a specific effect as: 1) such relationship was weak or inconsistent 527 between different analytical approaches for HPC theta power; 2) acceleration cannot 528 be used for reliable HPC theta frequency and power predictions and; 3) identical 529 analyses carried out on SuM theta provided no evidence for kinematic scaling and 530 their relationship to water maze learning. Thus, we conclude that HPC theta 531 frequency and speed correlations serve its theorised role (Burgess and O'Keefe, 532 2011) in providing a distance metric in path integration for spatial cognition. 


\section{Acknowledgments}

534 This work was supported by the Marsden Fund of New Zealand (UOO105) and New

535 Zealand Neurological Foundation (0024/PG/McNaughton).

536 Contributions

537 CKY conceived the study, carried out the analyses and part of the histology. MR

538 collected the behavioural, neurophysiological and histological data. CKY and NM

539 prepared the manuscript.

\section{References}

541

542

543

544

545

546

547

548

549

550

551

552

553

554

555

556

557

558

559

560

561

562

563

564

565

566

567

568

569

570

571

572

573

574

575

Aghajan ZM, Schuette P, Fields TA, Tran ME, Siddiqui SM, Hasulak NR, Tcheng TK, Eliashiv D, Mankin EA, Stern J, Fried I, Suthana N (2017) Theta Oscillations in the Human Medial Temporal Lobe during Real-World Ambulatory Movement. Curr Biol 27:3743-3751 e3743.

Ahmed OJ, Mehta MR (2012) Running speed alters the frequency of hippocampal gamma oscillations. J Neurosci 32:7373-7383.

Belchior H, Lopes-Dos-Santos V, Tort AB, Ribeiro S (2014) Increase in Hippocampal Theta Oscillations during Spatial Decision-Making. Hippocampus.

Bland $\mathrm{BH}$ (1986) The physiology and pharmacology of hippocampal formation theta rhythms. Prog Neurobiol 26:1-54.

Bland BH, Oddie SD (2001) Theta band oscillation and synchrony in the hippocampal formation and associated structures: the case for its role in sensorimotor integration. Behav Brain Res 127:119-136.

Bohbot VD, Copara MS, Gotman J, Ekstrom AD (2017) Low-frequency theta oscillations in the human hippocampus during real-world and virtual navigation. Nat Commun 8:14415.

Bokil H, Andrews P, Kulkarni JE, Mehta S, Mitra PP (2010) Chronux: a platform for analyzing neural signals. J Neurosci Methods 192:146-151.

Bolding KA, Ferbinteanu J, Fox SE, Muller RU (2019) Place cell firing cannot support navigation without intact septal circuits. Hippocampus.

Brandon MP, Bogaard AR, Libby CP, Connerney MA, Gupta K, Hasselmo ME (2011) Reduction of theta rhythm dissociates grid cell spatial periodicity from directional tuning. Science 332:595-599.

Brugge JF (1965) An Electrographic Study of the Hippocampus and Neocortex in Unrestrained Rats Following Septal Lesions. Electroencephalogr Clin Neurophysiol 18:36-44.

Burgess N, O'Keefe J (2011) Models of place and grid cell firing and theta rhythmicity. Curr Opin Neurobiol 21:734-744.

Bush D, Bisby JA, Bird CM, Gollwitzer S, Rodionov R, Diehl B, McEvoy AW, Walker MC, Burgess N (2017) Human hippocampal theta power indicates movement onset and distance travelled. Proc Natl Acad Sci U S A 114:12297-12302.

Buzsaki G (2002) Theta oscillations in the hippocampus. Neuron 33:325-340.

Buzsaki G (2005) Theta rhythm of navigation: link between path integration and landmark navigation, episodic and semantic memory. Hippocampus 15:827840. 
576 Campbell MG, Ocko SA, Mallory CS, Low IIC, Ganguli S, Giocomo LM (2018) Principles governing the integration of landmark and self-motion cues in entorhinal cortical codes for navigation. Nat Neurosci 21:1096-1106.

Czurko A, Hirase H, Csicsvari J, Buzsaki G (1999) Sustained activation of hippocampal pyramidal cells by 'space clamping' in a running wheel. Eur $\mathrm{J}$ Neurosci 11:344-352.

Ekstrom AD, Caplan JB, Ho E, Shattuck K, Fried I, Kahana MJ (2005) Human hippocampal theta activity during virtual navigation. Hippocampus 15:881889.

Gois Z, Tort ABL (2018) Characterizing Speed Cells in the Rat Hippocampus. Cell reports 25:1872-1884 e1874.

Goutagny R, Jackson J, Williams S (2009) Self-generated theta oscillations in the hippocampus. Nat Neurosci.

Green JD, Arduini AA (1954) Hippocampal electrical activity in arousal. J Neurophysiol 17:533-557.

Hernandez-Perez JJ, Gutierrez-Guzman BE, Olvera-Cortes ME (2016) Hippocampal strata theta oscillations change their frequency and coupling during spatial learning. Neuroscience 337:224-241.

Hinman JR, Penley SC, Long LL, Escabi MA, Chrobak JJ (2011) Septotemporal variation in dynamics of theta: speed and habituation. J Neurophysiol 105:2675-2686.

Hinman JR, Brandon MP, Climer JR, Chapman GW, Hasselmo ME (2016) Multiple Running Speed Signals in Medial Entorhinal Cortex. Neuron 91:666-679.

Hollup SA, Molden S, Donnett JG, Moser MB, Moser El (2001a) Accumulation of hippocampal place fields at the goal location in an annular watermaze task. $\mathrm{J}$ Neurosci 21:1635-1644.

Hollup SA, Molden S, Donnett JG, Moser MB, Moser El (2001b) Place fields of rat hippocampal pyramidal cells and spatial learning in the watermaze. Eur $\mathrm{J}$ Neurosci 13:1197-1208.

Jayakumar RP, Madhav MS, Savelli F, Blair HT, Cowan NJ, Knierim JJ (2019) Recalibration of path integration in hippocampal place cells. Nature 566:533537.

Jeewajee A, Lever C, Burton S, O'Keefe J, Burgess N (2007) Environmental novelty is signaled by reduction of the hippocampal theta frequency. Hippocampus.

Jeewajee A, Lever C, Burton S, O'Keefe J, Burgess N (2008) Environmental novelty is signaled by reduction of the hippocampal theta frequency. Hippocampus 18:340-348.

King C, Recce M, O'Keefe J (1998) The rhythmicity of cells of the medial septum/diagonal band of Broca in the awake freely moving rat: relationships with behaviour and hippocampal theta. Eur J Neurosci 10:464-477.

Koenig J, Linder AN, Leutgeb JK, Leutgeb S (2011) The spatial periodicity of grid cells is not sustained during reduced theta oscillations. Science 332:592-595.

Korotkova T, Ponomarenko A, Monaghan CK, Poulter SL, Cacucci F, Wills T, Hasselmo ME, Lever C (2018) Reconciling the different faces of hippocampal theta: The role of theta oscillations in cognitive, emotional and innate behaviors. Neurosci Biobehav Rev 85:65-80.

Kropff E, Carmichael JE, Moser MB, Moser El (2015) Speed cells in the medial entorhinal cortex. Nature 523:419-424. 
624

625

626

627

628

629

630

631

632

633

634

635

636

637

638

639

640

641

642

643

644

645

646

647

648

649

650

651

652

653

654

655

656

657

658

659

660

661

662

663

664

665

666

667

668

669

670

671

672

673
Kunz L, Maidenbaum S, Chen D, Wang L, Jacobs J, Axmacher N (2019) Mesoscopic Neural Representations in Spatial Navigation. Trends Cogn Sci 23:615-630.

Kuo TB, Li JY, Chen CY, Yang CC (2011) Changes in hippocampal theta activity during initiation and maintenance of running in the rat. Neuroscience 194:2735.

Long LL, Hinman JR, Chen CM, Escabi MA, Chrobak JJ (2014) Theta Dynamics in Rat: Speed and Acceleration across the Septotemporal Axis. PLoS One 9:e97987.

Maurer AP, Vanrhoads SR, Sutherland GR, Lipa P, McNaughton BL (2005) Selfmotion and the origin of differential spatial scaling along the septo-temporal axis of the hippocampus. Hippocampus 15:841-852.

McFarland WL, Teitelbaum H, Hedges EK (1975) Relationship between hippocampal theta activity and running speed in the rat. Journal of comparative and physiological psychology 88:324-328.

McNaughton N, Ruan M, Woodnorth MA (2006) Restoring theta-like rhythmicity in rats restores initial learning in the Morris water maze. Hippocampus 16:11021110.

Montgomery SM, Betancur MI, Buzsaki G (2009) Behavior-dependent coordination of multiple theta dipoles in the hippocampus. J Neurosci 29:1381-1394.

Pan WX, McNaughton N (1997) The medial supramammillary nucleus, spatial learning and the frequency of hippocampal theta activity. Brain Res 764:101108.

Ravassard P, Kees A, Willers B, Ho D, Aharoni DA, Cushman J, Aghajan ZM, Mehta MR (2013) Multisensory control of hippocampal spatiotemporal selectivity. Science 340:1342-1346.

Rawlins JN, Feldon J, Gray JA (1979) Septo-hippocampal connections and the hippocampal theta rhythm. Exp Brain Res 37:49-63.

Richard GR, Titiz A, Tyler A, Holmes GL, Scott RC, Lenck-Santini PP (2013) Speed modulation of hippocampal theta frequency correlates with spatial memory performance. Hippocampus.

Rivas J, Gaztelu JM, Garcia-Austt E (1996) Changes in hippocampal cell discharge patterns and theta rhythm spectral properties as a function of walking velocity in the guinea pig. Exp Brain Res 108:113-118.

Ruan M, Young CK, McNaughton N (2017) Bi-Directional Theta Modulation between the Septo-Hippocampal System and the Mammillary Area in Free-Moving Rats. Front Neural Circuits 11:62.

Rutishauser U, Ross IB, Mamelak AN, Schuman EM (2010) Human memory strength is predicted by theta-frequency phase-locking of single neurons. Nature 464:903-907.

Schmidt B, Hinman JR, Jacobson TK, Szkudlarek E, Argraves M, Escabi MA, Markus EJ (2013) Dissociation between dorsal and ventral hippocampal theta oscillations during decision-making. J Neurosci 33:6212-6224.

Shin J, Talnov A (2001) A single trial analysis of hippocampal theta frequency during nonsteady wheel running in rats. Brain Res 897:217-221.

Shirvalkar PR, Rapp PR, Shapiro ML (2010) Bidirectional changes to hippocampal theta-gamma comodulation predict memory for recent spatial episodes. Proc Natl Acad Sci U S A 107:7054-7059.

Slawinska U, Kasicki S (1998) The frequency of rat's hippocampal theta rhythm is related to the speed of locomotion. Brain Res 796:327-331. 
674 Terrazas A, Krause M, Lipa P, Gothard KM, Barnes CA, McNaughton BL (2005)

675 Self-motion and the hippocampal spatial metric. J Neurosci 25:8085-8096.

676 Vanderwolf $\mathrm{CH}$ (1969) Hippocampal electrical activity and voluntary movement in the

677 rat. Electroencephalogr Clin Neurophysiol 26:407-418.

678 Watrous AJ, Fried I, Ekstrom AD (2011) Behavioral correlates of human

679

680

681

682 hippocampal delta and theta oscillations during navigation. J Neurophysiol 105:1747-1755.

Wells CE, Amos DP, Jeewajee A, Douchamps V, Rodgers J, O'Keefe J, Burgess N, Lever C (2013) Novelty and anxiolytic drugs dissociate two components of hippocampal theta in behaving rats. J Neurosci 33:8650-8667.

Whishaw IQ, Vanderwolf CH (1971) Hippocampal EEG and behavior: effects of variation in body temperature and relation of EEG to vibrissae movement, swimming and shivering. Physiol Behav 6:391-397.

Whishaw IQ, Vanderwolf CH (1973) Hippocampal EEG and behavior: changes in amplitude and frequency of RSA (theta rhythm) associated with spontaneous and learned movement patterns in rats and cats. Behavioral biology 8:461484.

Winson J (1978) Loss of hippocampal theta rhythm results in spatial memory deficit in the rat. Science 201:160-163.

Young CK (2011) Behavioral significance of hippocampal theta oscillations: looking elsewhere to find the right answers. J Neurophysiol 106:497-499.

Young CK, Eggermont JJ (2009) Coupling of mesoscopic brain oscillations: Recent advances in analytical and theoretical perspectives. Progress in Neurobiology 89:61-78.

Young CK, McNaughton N (2020) Mixed Effects of Low-dose Ethanol on Cortical and Hippocampal Theta Oscillations. Neuroscience 429:213-224.

700 
702 Table 1. Summary from Figures 4B, 4D, 5B and 5D. $r^{2}$ with $p<.05$ are highlighted in

703 bold. Hz: theta frequency; zEnv: theta power.

\begin{tabular}{l|r|r|r|r}
$\mathrm{Hz}$ & \multicolumn{2}{|l|}{ HPC } & \multicolumn{2}{l}{ SuM } \\
& \multicolumn{1}{l|}{ slope } & \multicolumn{1}{l}{$r^{2}$} & slope & $r^{2}$ \\
\hline speed & $\mathbf{0 . 5 4 2 5}$ & $\mathbf{0 . 7 1 4 6}$ & 0.218 & 0.4276 \\
accel. & 0.0127 & 0.369 & $\mathbf{0 . 6 5 4 5}$ & $\mathbf{0 . 5 6 0 7}$
\end{tabular}

\begin{tabular}{l|r|r|r|r} 
zEnv & \multicolumn{2}{|l|}{ HPC } & \multicolumn{2}{l}{ SuM } \\
& slope & $r^{2}$ & slope & $r^{2}$ \\
\hline speed & 0.0069 & 0.0041 & 0.234 & 0.2556 \\
accel. & 0.0477 & 0 & 0.0907 & 0.0204
\end{tabular}

705 Table 2. Summary of overall, positive (+ve) and negative (-ve) acceleration fits with 706 theta variables relating to Fig. 5. $r^{2}$ with $p<.05$ are highlighted in bold. $\mathrm{Hz}$ : theta

707 frequency; zEnv: theta power.

\begin{tabular}{|c|c|c|c|c|c|}
\hline $\mathrm{Hz}$ & $\begin{array}{l}\text { HPC } \\
r^{2}\end{array}$ & $\begin{array}{l}\text { SuM } \\
r^{2}\end{array}$ & zEnv & $\begin{array}{l}\text { HPC } \\
r^{2}\end{array}$ & $\begin{array}{l}\text { SuM } \\
r^{2}\end{array}$ \\
\hline overall & 0.6773 & 0.2442 & overall & 0.0085 & 0.0573 \\
\hline +ve & 0.0211 & 0.471 & +ve & 0.9765 & 0.3958 \\
\hline -ve & 0.792 & 0.1917 & -ve & 0.6163 & 0.8544 \\
\hline
\end{tabular}




\section{$710 \quad$ Figures}
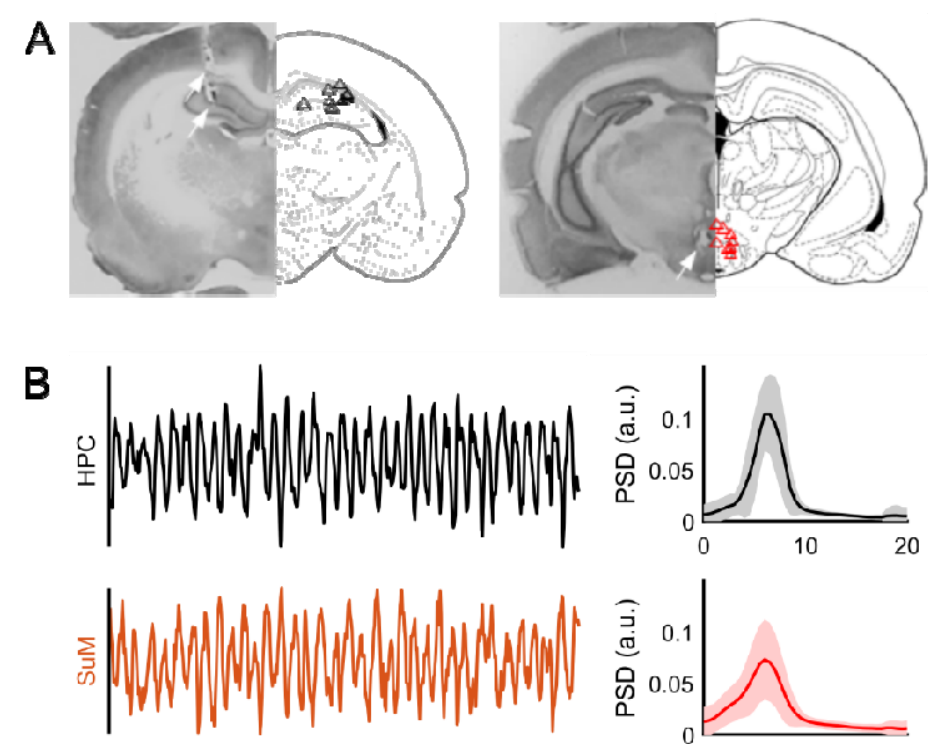

711

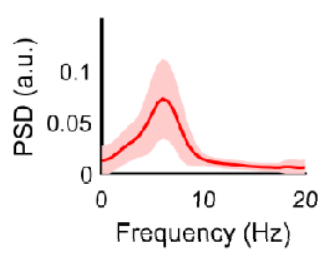

712 Figure 1. Theta field potential recordings. A: Example histology and reconstructed

713 recording locations in the hippocampus (left) and the supramammillary area. B: Field

714 potentials in the hippocampus (top, black) and the supramammillary area (bottom, 715 red) show continuous theta oscillations during swimming. 


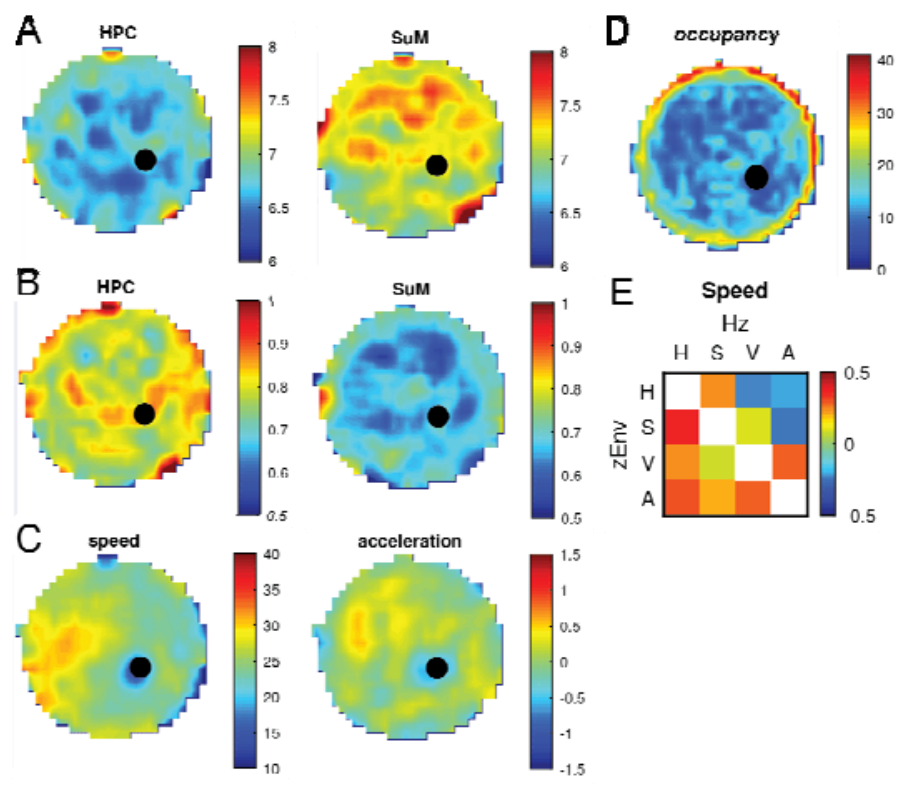

719 Figure 2. Spatial distribution of theta and kinematic variables in the water maze. A:

720 Averaged theta frequency distribution within the water maze in the hippocampus 721 (left) and the supramammillary area (right). B: Same as in A but for normalised 722 power. C: Averaged swimming speed (left) and acceleration (right) distributions 723 within the water maze. D: Occupancy map in the water maze. E: Spatial cross724 correlation matrix with frequency $(\mathrm{Hz})$ based cross-correlations on the top left half of 725 the matrix and power (zEnv) based cross-correlations on the bottom right half. $\mathrm{H}$ : 726 hippocampus; $M$ : supramammillary area; $\mathrm{V}$ : speed; $A$ : acceleration. Color maps ${ }^{2}$ 727 from -0.5 to 0.5 . 

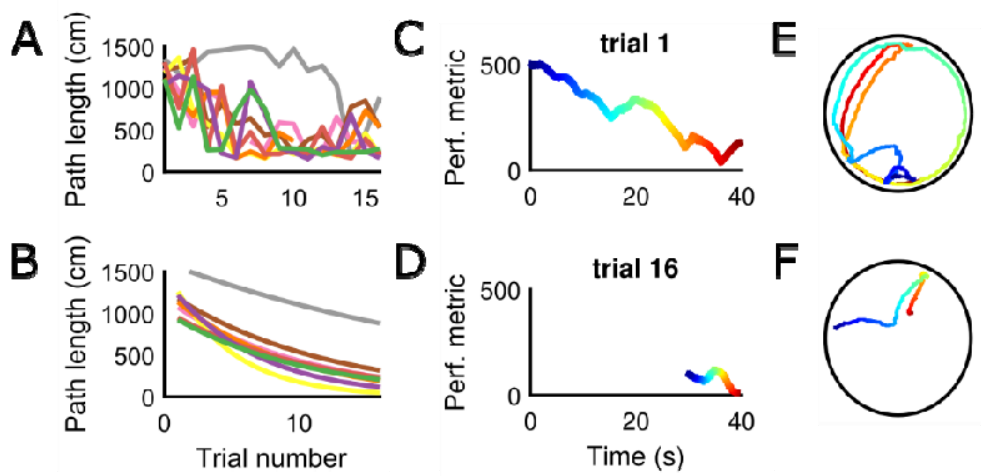

730 Figure 3. Characterisation of learning metrics in the water maze. A: Change of path

731 length across trials for all rats. B: Associated exponential fits for A. C: An example of 732 the water maze performance metric for the first exposure to the water maze. D: An 733 example of the water maze performance metric in the last trial. E: Actual swim path 734 in the water maze with color mapping to $\mathbf{C}$. F: Same as in $\mathbf{E}$ but for $\mathbf{D}$. 

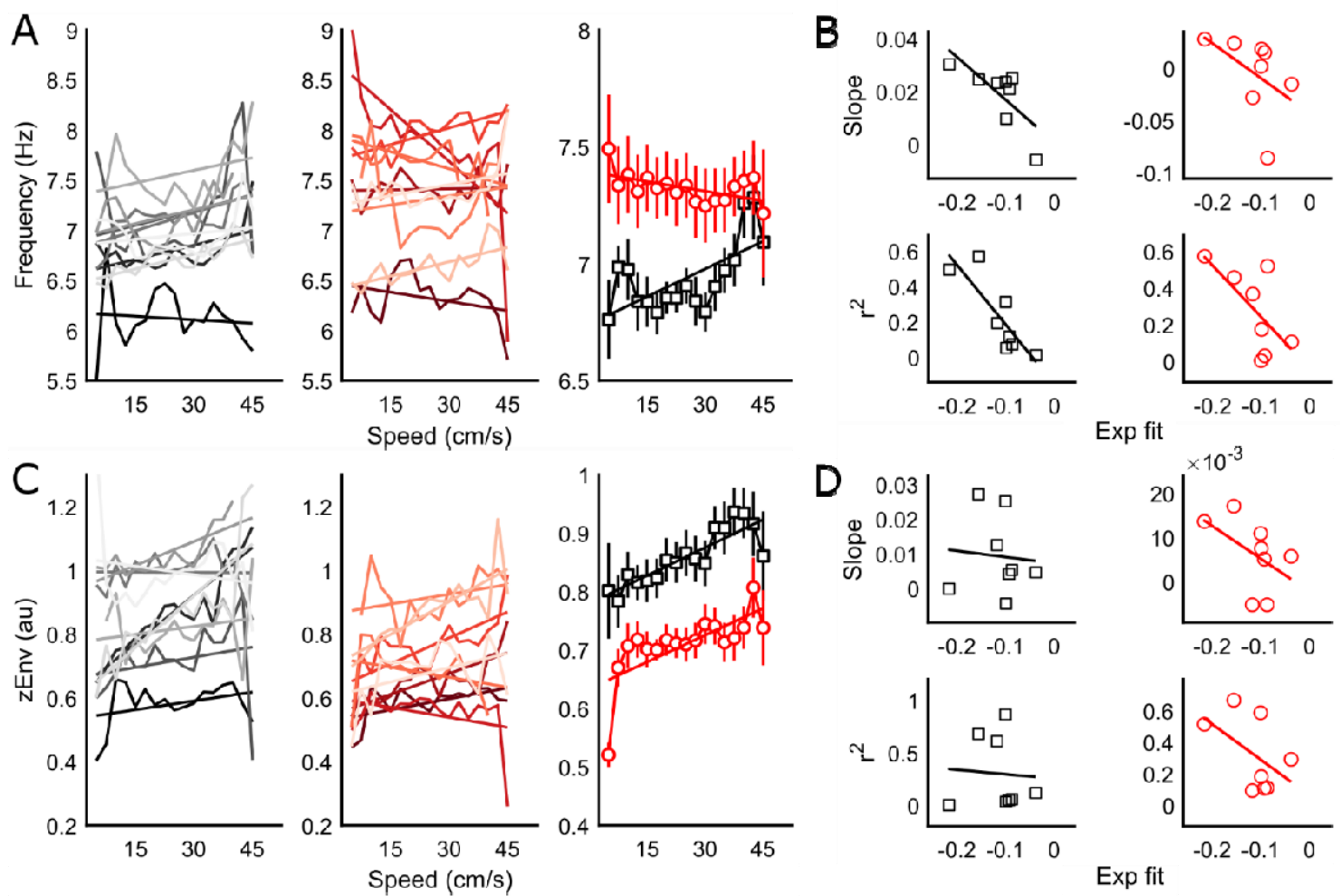

738

Figure 4. Speed modulation of hippocampal and supramammillary theta oscillations.

A: Raw data and their linear fits for the hippocampus (right, black) and the supramammillary area (middle, red) theta frequency across binned speeds. Population data and their fits are depicted in the right panel. B. Correlations between

742 the magnitude (slope, top) and robustness ( $r^{2}$, bottom) of the frequency versus

743 speed scaling and water maze learning (exponential fit slope parameter) for

744 hippocampal (black, left) and supramammillary (red, right) theta. C: Same as in A but 745 for speed versus theta power correlations. D: Same as in B but for theta power 746 versus speed scaling and water maze performance correlations. 

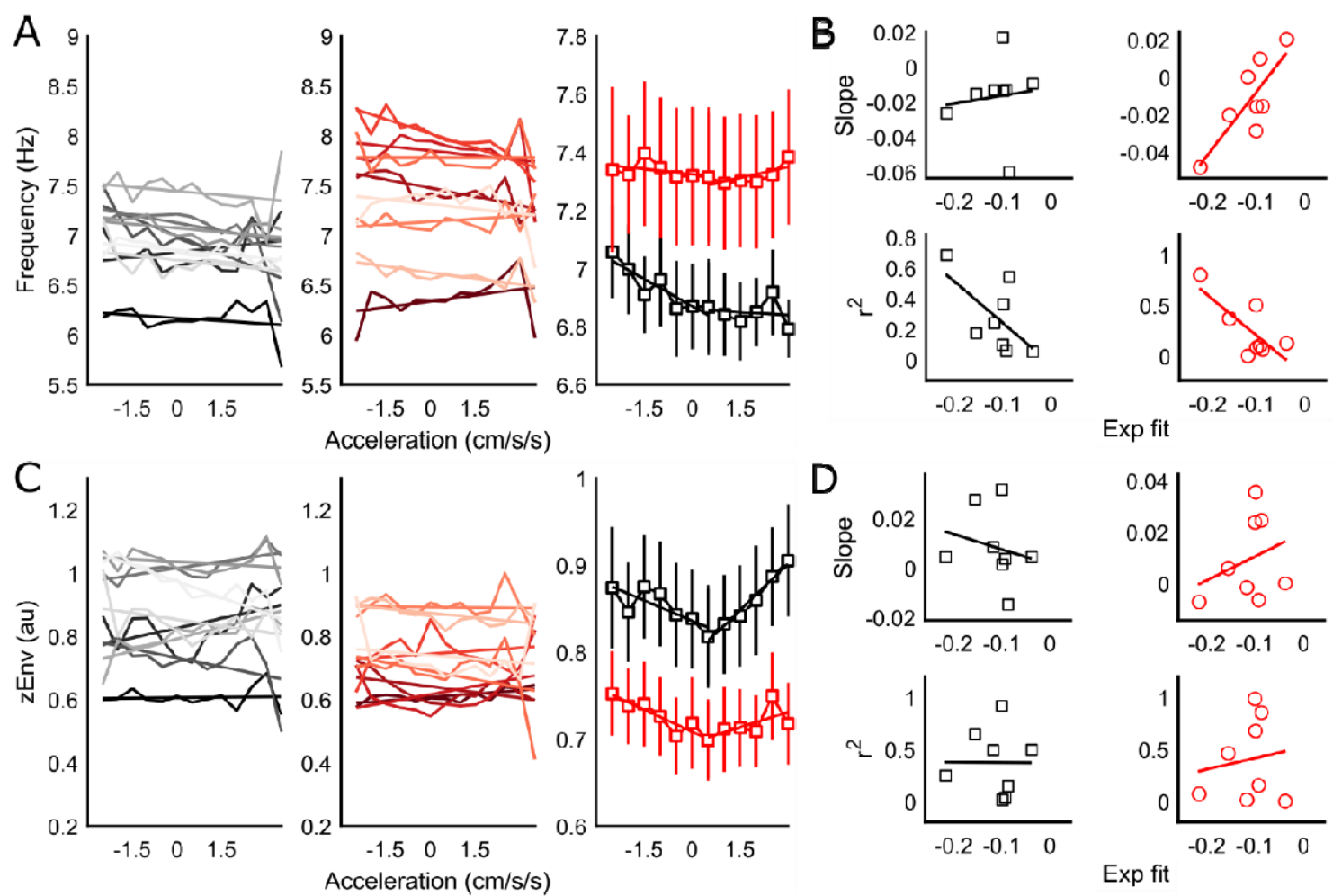

748 Figure 5. Acceleration modulation of hippocampal and supramammillary theta

749 oscillations. A: Raw data and their linear fits for the hippocampus (right, black) and

750 the supramammillary area (middle, red) theta frequency across binned acceleration.

751 Population data and their fits are depicted in the right panel. B. Correlations between

752 the magnitude (slope, top) and robustness ( $r^{2}$, bottom) of the frequency versus

753 acceleration scaling and water maze learning (exponential fit slope parameter) for

754 hippocampal (black, left) and supramammillary (red, right) theta. C: Same as in $\mathbf{A}$ but

755 for acceleration versus theta power correlations. D: Same as in B but for theta power

756 versus acceleration scaling and water maze performance correlations. 

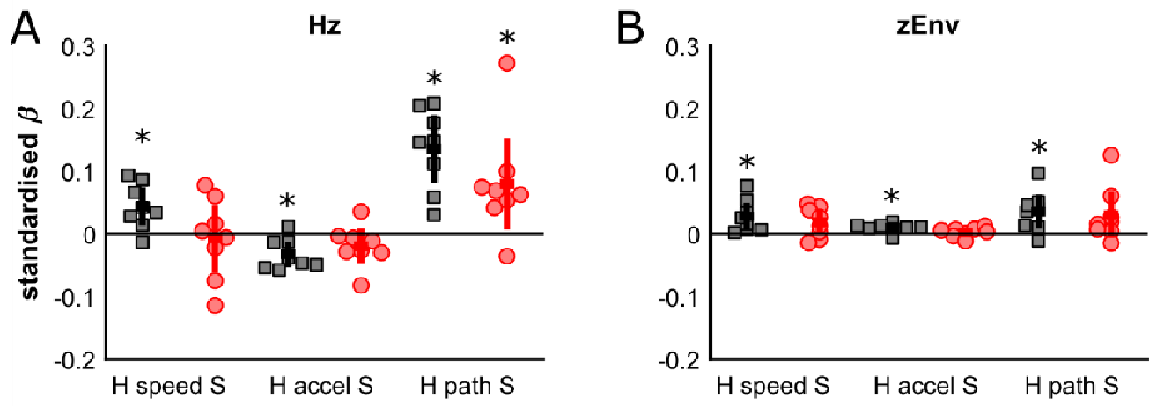

760 Figure 6. Standardised betas from GLM. A: Betas from each predictor variable from

761 each brain structure for theta frequency. B. Same as $\mathbf{A}$ but for theta power. $\mathrm{H}$ :

762 hippocampus; S: supramammillary area. Asterix denotes $p<.05$ with Student's t-

763 tests. 